\title{
Recém-Nascido de Muito Baixo Peso numa Unidade de Cuidados Intensivos Portuguesa Comparativamente ao Vermont Oxford Network: 15 Anos de Registo
}

\section{Very Low Birth Weight Infants in a Portuguese Intensive Care Unit and the Vermont Oxford Network: 15 Years of Registry Data}

\author{
Joana SALDANHA $\triangle^{1,2}$, Carlos MONIZ1,2, Maria do Céu MACHADO ${ }^{1,2}$ \\ Acta Med Port 2019 Nov;32(11):686-692 - https://doi.org/10.20344/amp.9130
}

\section{RESUMO}

Introdução: O nosso serviço de Neonatologia está integrado num hospital perinatal diferenciado e pertence à rede de registo Vermont Oxford Network desde há mais de 15 anos. Este registo inclui dados da morbi-mortalidade de recém-nascidos com peso de nascimento entre 401 e $1500 \mathrm{~g}$ e/ou das 22 às 29 semanas e seis dias de gestação, nascidos nos hospitais membros ou admitidos até aos 28 dias de vida. Permite a análise da prática clínica e comparação com unidades semelhantes. Foi nosso objetivo divulgar alguns dos nossos dados dos últimos 15 anos fazer a sua reflexão, o estudo dos resultados e evolução das práticas assistenciais neonatais ao longo dos anos, e compará-los com os dados de um grupo com o mesmo nível de cuidados da rede Vermont Oxford Network. Dado considerarmos ser fundamental o estudo dos dados de morbimortalidade das unidades de neonatologia e sua comparação com unidades congéneres no sentido de identificação de áreas suscetíveis de intervenção, consideramos a pertinência da divulgação dos nossos dados.

Material e Métodos: Estudo observacional, retrospetivo. Incluídos recém-nascidos com peso de nascimento $\leq 1500 \mathrm{~g}$ (recém-nascido de muito baixo peso) nascidos e tratados no nosso Hospital de 2001 a 2015 e comparados em dois subgrupos temporais com os dados da rede Vermont Oxford Network. Análise dos dados descritiva, teste de qui-quadrado e ANOVA, significância quando $p<0,05$. Resultados: Estudaram-se 869 recém-nascidos de muito baixo peso com uma mediana de peso $1100 \mathrm{~g}$ e idade gestacional 29 semanas. Eram gémeos $37,6 \%$. Na sala de partos $23 \%$ não necessitaram de qualquer reanimação, precisaram de entubação endotraqueal $52,2 \%$ dos recém-nascidos, em $78,3 \%$ foi administrado surfactante e desde que tal começou a ser registado em $2011,29,7 \%$ iniciaram de imediato ventilação não invasiva. Em relação às principais morbilidades estudadas do ponto de vista respiratório do total de recém-nascidos de muito baixo peso 12,9\% tinham oxigenoterapia às 36 semanas de idade corrigida, em relação à persistência do canal arterial hemodinamicamente significativo esta verificou-se em $23 \%$ e do ponto de vista infecioso verificou-se sépsis tardia em $17,1 \%$. Registámos maior morbilidade neurológica comparativamente à Vermont Oxford Network exceto na retinopatia da prematuridade. A mortalidade global foi de $14 \%$ (122 recém-nascidos). O tempo de internamento foi em média de $52,7 \pm 34,4$ dias. Os 629 recém-nascidos que tiveram alta para o domicílio estiveram internados sensivelmente os mesmos dias e apresentavam valores semelhantes de perímetro cefálico, mas menor peso no dia da alta que os da rede Vermont Oxford Network, tendo tido alta da nossa unidade com aleitamento materno exclusivo $14,3 \%$ dos recém-nascidos de muito baixo peso.

Discussão: Este trabalho permitiu fazer a reflexão sobre os dados do nosso serviço de Neonatologia e compará-los com os de um dos maiores registos neonatais mundiais. Verificámos que a nossa população de recém-nascidos de muito baixo peso é muito sobreponível do ponto de vista da idade gestacional e somatométrico, taxas de vigilância da gravidez e de cesarianas, sendo a diferença mais notória a percentagem de leves para a idade gestacional, de gestações gemelares e de realização de indução maturativa que foram superiores no nosso centro. As patologias do foro cardiorrespiratório e gastrointestinais foram sobreponíveis. Verificámos que é urgente melhorar a taxa de infeção associada aos cuidados de saúde, das sequelas neurológicas, do controle da hipotermia após o nascimento e da neuroprotecção com sulfato de magnésio. A taxa de mortalidade foi sobreponível assim como o tempo de internamento. Conclusão: Este trabalho permitiu-nos comparar a nossa população de recém-nascidos de muito baixo peso com os registados na rede. Verificámos que acompanhámos a evolução que a Neonatologia foi tendo ao longo dos anos e identificámos áreas suscetíveis de melhoria.

Palavras-chave: Benchmarking; Mortalidade Infantil; Recém-Nascido de Peso Extremamente Baixo ao Nascer; Recém-Nascido de Muito Baixo Peso; Unidades de Cuidados Intensivos Neonatais

\section{ABSTRACT}

Introduction: Our neonatal service is part of a differentiated perinatal hospital and has contributed to the Vermont Oxford Network for more than 15 years. This data base includes data on the morbidity and mortality of newborns born in the member hospitals with birth weight between 401 and $1500 \mathrm{~g}$ and/ or from 22 to 29 weeks and six days of gestation, or those admitted to these hospitals with up to 28 days of age. It thus allows the analysis of clinical practice and its comparison with similar units. The goal of the present paper is to disclose some of our data from the past 15 years and to compare it with the Vermont Oxford Network data trying to identify areas of possible improvement and permitting other neonatal units to compare their data with our in a benchmarking process.

Material and Methods: Observational, retrospective study. It included newborns with birth weight $\leq 1500 \mathrm{~g}$ (very low birth weight newborns) born and treated at our hospital from 2001 to 2015. Descriptive data analysis, chi-square test and ANOVA, significance when $p<0.05$.

1. Serviço de Neonatologia. Departamento de Pediatria. Hospital de Santa Maria. Centro Hospitalar Lisboa Norte. Lisboa. Portugal.

2. Centro Académico de Lisboa. Lisboa. Portugal.

$\triangle$ Autor correspondente: Joana Saldanha. joanasaldanha@sapo.pt

Recebido: 26 de abril de 2017 - Aceite: 14 de maio de 2019 | Copyright @ Ordem dos Médicos 2019 
Results: A total of 869 very low birth weight newborns were studied, median weight $1100 \mathrm{~g}$ and gestational age 29 weeks. Twinning was found in $37.6 \%$. In the delivery room, $23 \%$ did not require any resuscitation, $52.2 \%$ of the newborns required invasive intubation, $78.3 \%$ had surfactant, and, since $2011,29.7 \%$ have started noninvasive ventilation. Of the total very low birth weight newborns, $12.9 \%$ had oxygen therapy at 36 weeks of corrected age, $23 \%$ patent ductus arteriosus and late sepsis in $17.1 \%$. There was higher neurological morbidity compared to the Vermont Oxford Network except in the case of retinopathy of prematurity. Overall mortality was $14 \%$ (122 newborns). The time of hospitalization was on average $52.7 \pm 34.4$ days. The 629 newborns that were discharged home had equivalent length of stay and head circumference measure but a lower weight than those in the Vermont Oxford Network, and $14.3 \%$ went home with exclusive breastfeeding.

Discussion: This work allowed us to study our very low birth weight newborns data and compare it with one of the largest neonatal world networks. Our population is similar from the point of view of gestational age, somatometric data, pregnancy surveillance rates and cesarean section with the most noticeable difference being the percentage of low birthweight for gestational age babies, twin pregnancies and antenatal corticosteroid treatment, superior in our center. Cardio-pulmonary and gastrointestinal disorders were overlapping. It is urgent to improve our rate of sepsis, neurologic sequelae, post-partum hypothermia control and neuroprotection with magnesium sulphate. The mortality rate and the length of stay at discharge was similar.

Conclusion: This study allowed us to compare our population of very low birth weight newborns with those registered in the network. We have verified that we have been accompanying the evolution of Neonatology over the past years and we have identified areas for improvement.

Keywords: Benchmarking; Infant, Extremely Low Birth Weight; Infant Mortality; Infant, Very Low Birth Weight; Intensive Care Units, Neonatal

\section{INTRODUÇÃO}

A evolução técnica e científica da Neonatologia ao longo dos anos tem permitido a diminuição do limiar da viabilidade e uma maior sobrevida com menor morbilidade. O conceito de medicina perinatal permitiu uma generalização da indução maturativa abaixo das 34 semanas assim como a tentativa de adiamento até o mais tarde possível dos partos muito prematuros e a sua transferência in utero para hospitais perinatais diferenciados.

$\mathrm{O}$ registo de dados da Vermont Oxford Network (V/O) é uma comunidade de profissionais de saúde que colaboram na otimização da qualidade e segurança dos cuidados médicos prestados a recém-nascidos (RN) e suas famílias através de programas de investigação e de educação, e de projetos de melhoria da qualidade dos cuidados peri e neonatais. A rede, sediada nos Estados Unidos da América, teve início em 1988, conta atualmente com quase 1000 Unidades de Cuidados Intensivos Neonatais (UCIN) americanas e de 28 países de outros continentes (das quais 193 UCIN europeias). Mantém e estuda bases de registo de dados que incluem informação acerca da morbi-mortalidade dos recém-nascidos e cuidados prestados nas UCIN dos membros participantes. São registados recém-nascidos, com peso de nascimento entre 401 e 1500 g [recém-nascido de muito baixo peso (RNMBP)] e/ou das 22 às 29 semanas e seis dias, nascidos em cada hospital ou admitidos até aos 28 dias de vida. A rede V/O tem já registados dados de mais de dois milhões de recém-nascidos representando mais de 63 milhões de dias de internamento de doentes. ${ }^{1-3}$

Pretende-se que cada unidade participante possa, através de um estudo comparativo com os dados da rede, melhorar resultados. A rede promove ainda estudos multicêntricos, aleatorizados procurando respostas para vários problemas perinatais. A nossa UCIN participou há uns anos no HelP trial que tinha como objetivo estudar se a evicção da hipotermia logo após o nascimento alteraria a mortalidade e morbilidade. ${ }^{4,5}$ Foi muito interessante a participação neste estudo internacional, que levou a melhorar práticas assistenciais na nossa UCIN.
O serviço de Neonatologia do nosso hospital perinatal diferenciado participa na rede desde 2001 e avalia regularmente os dados nomeadamente estudando desvios ou resultados fora da média dos dados globais, permitindo identificar áreas suscetíveis de intervenção.

Este trabalho tem como objetivo divulgar os dados da UCIN de um hospital perinatal diferenciado português, colhidos durante 15 anos, sua reflexão, o estudo da evolução de diferentes práticas assistenciais neonatais ao longo dos anos, e comparação com os resultados do grupo com o mesmo nível de cuidados da rede $\mathrm{V} / \mathrm{O}$ na sua globalidade e temporalmente. Outras UCIN poderão ainda ter interesse em fazer o estudo comparativo com os seus próprios dados.

\section{MATERIAL E MÉTODOS}

Estudo observacional, retrospectivo. Foram estudados os recém-nascidos com peso de nascimento (PN) inferior ou igual a $1500 \mathrm{~g}$ (RNMBP) nascidos e tratados no nosso hospital durante um período de 15 anos, de 2001 a 2015, quer na sua totalidade, quer separados em dois grupos temporais de 2001 a 2008 (G01-08) e de 2009 a 2015 (G09-15). Os dados estudados foram os dados da gravidez (vigilância, indução maturativa pré-natal, administração de sulfato de magnésio, gemelaridade, patologia hipertensiva e corioamnionite), tipo de parto, características dos recémnascidos (idade gestacional, peso (PN) e perímetro cefálico (PC) ao nascer, sexo, leve para a idade gestacional) tipo de reanimação na sala de partos, índice de Apgar ao primeiro e quinto minutos, administração de surfactante na sala de partos, temperatura na admissão à UCIN, tipo de apoio respiratório na UCIN, morbilidade respiratória, cardíaca, infeciosa, do sistema nervoso central - SNC (utilização da classificação de Papile et $a l^{6}$ com quatro graus de gravidade para as hemorragias intra e peri ventriculares (HIPV), oftalmológica, gastrointestinal e malformativa, dados da alta (para o domicilio, transferência para outro hospital ou óbito, peso e PC na alta, dias de internamento na UCIN do estudo). Para a classificação de leve para a idade gestacional (LIG) a rede de V/O utiliza as US Vital Statistics Natality 
data sets classificando de LIG os RN abaixo do percentil 10 para o $\mathrm{PN}$, raça materna e sexo.

Foram excluídos os registos de RN que não correspondessem a estes critérios.

As unidades pertencentes à rede $\mathrm{V} / \mathrm{O}$ dividem-se em três tipos. O nosso centro hospitalar inclui-se no subgrupo $\mathrm{B}$ que presta todos os cuidados neonatais, incluindo qualquer tipo de ventilação, utilização de óxido nítrico e cirurgia neonatal exceto a cardíaca, sendo considerada de apoio perinatal diferenciado. As unidades de tipo A são consideradas de cuidados intermédios ou de apoio perinatal sem capacidade para proporcionar ventilação de longa duração ou outros cuidados neonatais mais intensivos, e as unidades de tipo $C$ são aquelas em que existe capacidade para realização de cirurgias cardíacas com circulação extra-corporal. Alguns dos dados de cada grupo foram também comparados com os dados dos recémnascidos do subgrupo $\mathrm{B}$ da rede $\mathrm{V} / \mathrm{O}$ nascidos em cada hospital de tratamento (inborn) nos anos de 2008 e 2015, respetivamente.

Utilizando a base de dados SPSS da IBM Statistics versão 21 , foi feita a análise descritiva dos dados. Para as variáveis continuas encontrada a média, desvio padrão e mediana. Para análise das variáveis categóricas as frequências e percentagens e utilizado o teste de qui-quadrado e exato de Fisher sempre que necessário e ANOVA. Foi considerado significância estatística quando $p<0,05$.

$O$ registo dos recém-nascidos da UCIN na base de dados Vermont/Oxford está autorizado pela Comissão de Ética do Centro Hospitalar, sendo este atualmente o financiador do registo pelo que não foi necessário obter autorização específica para este estudo em particular.

\section{RESULTADOS}

Em 15 anos foram registados pela nossa UCIN 952 RN, dos quais $63(6,6 \%)$ transferidos de outros hospitais. Faleceram na sala de partos cinco $(0,5 \%)$ e nove $(0,9 \%)$ tinham mais de $1500 \mathrm{~g}$ de PN. Foram transferidos para fora do hospital por falta de vaga na UCIN nas primeiras horas de vida seis RN (0,6\%). Assim, verificou-se que 869 RNMBP nasceram e foram tratados no hospital, e foram estes que estudámos.
Nos anos referentes ao G01-08 foram registados 451 RNMBP e no G09-15 registados 418. No subgrupo B do V/O em 2008 e em 2015 encontrámos respetivamente 27 649 e 26373 recém-nascidos.

A média anual de internamentos na nossa UCIN foi de 58 RNMBP, variando entre um mínimo de 44 e um máximo de 70 .

As características da gravidez, parto e dos RN divididos por grupos temporais na nossa unidade e no V/O são as descritas na Tabela 1. O peso mínimo de nascimento foi no G01-08 de $390 \mathrm{~g}$ e no G09-15 de $300 \mathrm{~g}$. No global de todos os RN em 15 anos verificou-se que a média dos pesos foi de $1068 \mathrm{~g}$ ( $\pm 280,2 \mathrm{~g}$ ) com uma mediana de $1100 \mathrm{~g}$. As idades gestacionais (IG) variaram entre $23 \mathrm{e}$ 38 semanas com uma média de 28,7 semanas $( \pm 2,7)$ e mediana de 29 semanas. Registámos somente dois RN de termo (38 semanas com grande restrição de crescimento intra-uterino), todos os outros incluídos neste estudo eram prematuros ( $\leq 36$ semanas de gestação). Em relação aos RN LIG verificámos na globalidade um valor de $28 \%$ do total dos nossos RN, enquanto na rede $\mathrm{V} / \mathrm{O}$ nos anos estudados foi em média de 23,3\%.

Tratámos 327 gémeos no total dos 15 anos (37,6\% dos $\mathrm{RN})$. Em 2005 iniciou-se o registo do número de gémeos e, num total de 643 RN, 198 eram fruto de gestação bigemelar e 38 trigemelar. Houve uma nítida diminuição dos partos trigemelares ao longo dos anos, mas não houve diferença estatisticamente significativa entre o número de gestações gemelares ao longo dos anos $(p=0,092)$ A partir de 2008 iniciou-se o registo das complicações da gravidez como corioamnionite e doença hipertensiva materna e num total de 474 RN, verificaram-se em $10,8 \%$ e $27,6 \%$ das gestações, respetivamente. A partir de 2012 iniciou-se o registo da administração pré-parto de sulfato de magnésio como neuro-protetor, nos nossos registos (de $239 \mathrm{RN}$ ) a sua administração foi apenas registada em 13,4\% dos casos enquanto na rede $\mathrm{V} / \mathrm{O}$ foi de $53,3 \%$.

As condições de reanimação na sala de partos (SP) assim como a necessidade de apoio respiratório na UCIN estão descritas na Tabela 2. A mediana do índice de Apgar (IA) ao primeiro minuto foi de 7 e ao quinto minuto de 9. Com um $I A \leq 4$ ao primeiro e quinto minutos registámos

Tabela 1 - Características dos recém-nascidos e dados da gravidez e tipo de parto

\begin{tabular}{|c|c|c|c|c|}
\hline & $\begin{array}{l}\mathrm{G} 01-08 \\
\mathrm{n}=451^{*}\end{array}$ & $\begin{array}{l}\text { V/O } 2008 \\
\mathrm{n}=27649\end{array}$ & $\begin{array}{c}G 09-15 \\
n=418\end{array}$ & $\begin{array}{l}\text { V/O } 2015 \\
n=26373\end{array}$ \\
\hline Peso (média g \pm desvio padrão/ mediana g) & $1069 \pm 283 / 1110$ & 1047 & $1067 \pm 276 / 1098$ & 1051 \\
\hline Idade gestacional (média g \pm desvio padrão/ mediana g) & $28,6 \pm 2,6 / 29$ & 28 & $28,8 \pm 2,7 / 29$ & 28 \\
\hline $\begin{array}{l}\text { Perímetro cefálico ao nascer (média g } \pm \text { desvio padrão/ } \\
\text { mediana g) }\end{array}$ & $25,5 \pm 2,3 / 25,8$ & 25,6 & $25,8 \pm 2,5 / 26$ & 25,6 \\
\hline Sexo masculino (\%) & 47,5 & 50,3 & 48,3 & 50,4 \\
\hline Gravidez vigiada (\%) & 94 & 96,1 & 94,5 & 96,5 \\
\hline Indução maturativa pré-natal (\%) & 81,6 & 78,3 & 92,3 & 84,9 \\
\hline Gémeos (\%) & 37,3 & 29,3 & 38 & 28,2 \\
\hline Cesariana (\%) & 77,6 & 72,6 & 74,2 & 73,7 \\
\hline
\end{tabular}

* Excepto perímetro cefálico em que $\mathrm{n}=149$ 
Tabela 2 - Reanimação na sala de partos e apoio respiratório na UCIN

\begin{tabular}{|c|c|c|c|c|}
\hline$\%$ & $\begin{array}{c}\text { G } 01-08 \\
n=451\end{array}$ & $\begin{array}{c}\text { V/O } 2008 \\
n=27649\end{array}$ & $\begin{array}{c}G 09-15 \\
n=418\end{array}$ & $\begin{array}{l}\text { V/O } 2015 \\
n=26373\end{array}$ \\
\hline Oxigénio sala partos & 77,4 & 85,9 & 71,5 & 82,5 \\
\hline Entubação traqueal sala partos & 58,1 & 50,4 & 45,9 & 42 \\
\hline nCPAP sala partos & na* & na* & 29,7 & 46,6 \\
\hline Surfactante sala partos & 38,4 & 32,4 & 54,5 & 25,3 \\
\hline Oxigénio UCIN & 79,4 & 89 & 79,4 & 85,9 \\
\hline Ventilação convencional na UCIN & 55,9 & 61,5 & 43,3 & 53,7 \\
\hline Ventilação alta frequência UCIN & 13,1 & 22,2 & 17,2 & 21,6 \\
\hline Qualquer tipo ventilação UCIN & 58,5 & 64,7 & 52,4 & 56,7 \\
\hline nCPAP UCIN $\neq$ & 69,6 & 68,8 & 81,3 & 77,8 \\
\hline nCPAP como $1^{a}$ abordagem & 30,4 & 41,5 & 46,2 & 62 \\
\hline nBiPAP§ ou outras formas de IMV nasal & 15,4 & 15 & 29,7 & 35,8 \\
\hline Alto fluxo por cânulas nasais & 13,4 & 48,7 & 25,6 & 53,5 \\
\hline
\end{tabular}

* na: não aplicável; ₹ nCPAP: nasal continuous positive airway pressure (registos na sala de partos a partir ano $2011 \mathrm{n}=296$ ); $\S \mathrm{nBiPAP}:$ nasal biphasic positive airway pressure; IMV: intermitent mandatory ventilation

respetivamente $17,8 \%$ e $2,9 \%$ dos RN. A entubação endotraqueal na reanimação aconteceu em 52,2\% dos RN havendo uma diminuição significativa com o passar dos anos $(p=0,000)$. Em 18 RN $(2,1 \%)$ foi necessário administrar adrenalina durante a reanimação e a 13 RN $(1,5 \%)$ fazer compressões torácicas. Verificámos ainda que 200 (23\%) RN não necessitaram de ser submetidos a qualquer reanimação nomeadamente administração de oxigénio.

$\mathrm{O}$ registo da utilização de nCPAP (nasal continuous positive airway pressure) na sala de partos iniciou-se em 2011, de 296 crianças 88 (29,7\%) iniciaram ventilação não invasiva na sala de partos e ao longo destes anos a sua utilização tem vindo a crescer significativamente $(p=0,03)$ com $42,6 \%$ dos RN em 2015 a serem colocados de imediato em nCPAP após o nascimento. A administração de surfactante profilático na SP foi crescendo, tendo atingido um pico em 2007 (78,3\% dos RN). Desde então decresceu, com administração em 39,3\% dos RN em 2015 ( $p<0,001)$. A medição da temperatura na primeira hora de admissão na UCIN começou a ser registada em 2006. Dos 585 RN, 383 cumpriram esse registo com uma média de temperatura na admissão de $35,29^{\circ} \mathrm{C}( \pm 0,8)$, mediana de $35,3^{\circ} \mathrm{C}$ $\left(35,0-35,5^{\circ} \mathrm{C}\right)$. Não houve diferenças significativas na temperatura de admissão ao longo dos anos $(p=0,2)$. Faleceram nas primeiras 12 horas de internamento na UCIN $17(2 \%)$ crianças.

Quanto à necessidade de apoio respiratório na UCIN, como se pode verificar na Tabela 2, assistimos ao longo destes anos à introdução de técnicas de ventilação não invasivas e aumento progressivo da sua utilização não só do nCPAP mas também do nBiPAP (nasal biphasic positive airway pressure/ nasal bilevel) (37,7\% dos RN em 2015) e a administração de alto fluxo por cânulas nasais de mistura variável de ar e oxigénio (62,3\% em 2015). A utilização de nCPAP como primeira abordagem ventilatória tem crescido mas ainda fica aquém da utilizada na rede V/O no ano de 2015 (62\%).
Quanto à morbilidade respiratória, obtivemos resultados sobreponíveis ou melhores que os da rede $\mathrm{V} / \mathrm{O}$, nomeadamente na percentagem de RNMBP com necessidade de oxigenoterapia às 36 semanas de idade corrigida (12,9\% do total). Quanto à alta para o domicílio com oxigénio $(3,3 \%)$ os nossos resultados divergem dos do $\mathrm{V} / \mathrm{O}$ possivelmente por diferentes políticas de alta na unidade. No entanto, a diferença entre os dois grupos temporais (de $0,9 \%$ para $5,7 \%$ ) pode traduzir uma mudança de abordagem na altura da alta. A utilização de corticosteroides como terapêutica adjuvante em caso de displasia broncopulmonar é residual (Tabela 3).

Em relação a patologia cardiovascular podemos verificar na Tabela 3 que os nossos doentes tiveram taxas de persistência do canal arterial (PCA) hemodinamicamente significativo e necessidade de terapêutica tendencialmente sobreponíveis aos RN da rede global. Dos 200 RNMBP ( $23 \%$ do total) com PCA, a média de IG foi de 26,7 semanas $\pm 1,9$ e mediana de 27 semanas e de PN de $898 \mathrm{~g} \pm 255$ com mediana de $867 \mathrm{~g}$, enquanto que nos sem PCA verificou-se uma IG média de 29,3 semanas $\pm 2,6$ com mediana de 29 e PN de $1118 \mathrm{~g} \pm 267$, mediana $1165 \mathrm{~g}(p<0,001)$. A existência de PCA associou-se significativamente ao menor PN, menor IG, necessidade de ventilação mecânica, oxigenoterapia às 36 semanas de IC, à existência de HIPV grave (graus 3 e 4), a existência de quadros de enterocolite necrosante (NEC), à maior mortalidade destes RNMBP (todos com $p<0,001$ ), e ao aparecimento de leucomalácea periventricular (LPV) $(p=0,002)$ e de retinopatia da prematuridade (ROP) grave (graus 3 e 4$)(p=0,001)$.

Em relação ao diagnóstico de sépsis (Tabela 3 ), só são incluídos no registo casos com isolamento bacteriológico ou fúngico, não sendo consideradas as sépsis clínicas. De $820 \mathrm{RN}$ ainda internados com mais de três dias de vida, $149(17,1 \%)$ tiveram sépsis considerada associada aos cuidados de saúde, verificando-se uma associação significativa entre maior mortalidade e sépsis $(p<0,001)$. As sépsis 
Tabela 3 - Morbilidade respiratória, cardíaca e infeciosa

\begin{tabular}{|c|c|c|c|c|}
\hline$\%$ & $\begin{array}{c}\text { G } 01-08 \\
n=451\end{array}$ & $\begin{array}{l}\text { V/O } 2008 \\
n=27649\end{array}$ & $\begin{array}{c}\text { G } 09-15 \\
n=418\end{array}$ & $\begin{array}{c}\text { V/O } 2015 \\
n=26273\end{array}$ \\
\hline $\mathrm{SDR}^{*}$ & 57,2 & 74,9 & 46,9 & 72,8 \\
\hline Pneumotórax & 4,9 & 4,4 & 5 & 4,1 \\
\hline CS DBP $\neq$ & 6,2 & 8,7 & 3,3 & 10,1 \\
\hline Oxigenoterapia às 28 semanas IC§ & 32,8 & 47,8 & 33 & 44,2 \\
\hline Oxigenoterapia às 36 semanas IC§ & 12,4 & 31,7 & 13,4 & 23,4 \\
\hline CPAP nasal às 36 semanas ICß & nall & nall & 0,2 & 6,4 \\
\hline Oxigenoterapia para domicílio & 0,9 & 12,4 & 5,7 & 11,8 \\
\hline PCA & 22,4 & 37,8 & 23,7 & 28 \\
\hline Indometacina ou ibuprofeno & 20,1 & 33 & 18,6 & 21,3 \\
\hline Cirurgia PCAף & 2,9 & 7,5 & 4,3 & 3,4 \\
\hline Sépsis precoce & 1,1 & 2,4 & 2,8 & 3,2 \\
\hline Sépsis bacteriana & 5,3 & 10,6 & 7,9 & 8,2 \\
\hline Sépsis estafilococo coagulase negativo & 5,8 & 10,8 & 19,1 & 5,5 \\
\hline Sépsis a fungos & 2,4 & 1,9 & 1 & 0,9 \\
\hline
\end{tabular}

*SDR: síndrome de dificuldade respiratória; \# CS DBP; corticoides para displasia broncopulmonar; § IC: idade corrigida; II na: não aplicável; \ PCA: persistência do canal arterial

precoces e por fungos foram identificadas num pequeno número de casos, sendo a sépsis por estafilococos coagulase negativos o maior problema infeccioso dos nossos RNMBP, com ocorrência significativa em idades gestacionais mais baixas $(p<0,001)$.

Como seria de esperar, a HIPV grave associou-se significativamente a uma maior mortalidade $(p<0,001)$.

A taxa de realização de ecografia transfontanelar nos primeiros 28 dias de vida foi de $97,1 \%$, tendo-se verificado um maior número percentual de HIPV grau 3 e 4 e LPV que no global da rede. Como seria de esperar a HIPV grave associou-se significativamente com a mortalidade $(p=0,000)$. A taxa de ROP grave foi semelhante aos dados globais do $\mathrm{V} / \mathrm{O}$, parecendo haver uma ligeira diminuição temporal (Tabela 4).

A patologia gastrointestinal e as malformações major encontradas foram as descritas na Tabela 5. Verificámos uma menor taxa de NEC no G01-08 (2,9\%) relativamente à totalidade dos 15 anos (4,1\%), não sendo muito diferente do global dos RN da rede $\mathrm{V} / \mathrm{O}$.

A mortalidade global no nosso hospital foi de $14 \%$ (122 RN), com ligeira diminuição, mas sem significância no segundo período estudado $(p=0,2)$. Se considerarmos só os RN com PN $\leq 1000 \mathrm{~g}$ (total de 344) a mortalidade foi de $25,3 \%$ e de $38,4 \%$ se considerarmos os 151 RNMBP com $\leq 750 \mathrm{~g}$.

Dos 869 RN, 664 tiveram alta para o domicílio,122 faleceram $(14 \%)$ e $83(9,5 \%)$ foram transferidos para outros hospitais, geralmente perto do local de residência da família para continuação de cuidados. O tempo de internamento na UCIN do estudo para todos os RN foi em média de $52,7 \pm 34,4$ dias (1 - 366 dias) com mediana de 48 dias, sendo para os sobreviventes em média de $59 \pm 30,8$ dias e mediana de 53 dias (alta para o domicilio com média de dias de internamento de $61 \pm 28$ dias e mediana de 55 dias e para os 83 transferidos para outros hospitais média de $39 \pm 26$ dias e mediana de 34 dias) e para os que faleceram média de 14,1 $\pm 30,44$ dias e mediana de seis dias. Os nossos RN tiveram na sua totalidade alta com aproximadamente os mesmos dias de internamento e valor de $\mathrm{PC}$, e com um peso sensivelmente menor que os da rede V/O. (Tabela 6).

O tipo de alimentação aquando da alta foi registado a partir de 2002. Com alta para o domicílio registámos desde

Tabela 4 - Patologia SNC e oftalmológica

\begin{tabular}{|c|c|c|c|c|}
\hline$\%$ & $\begin{array}{c}\text { G } 01-08 \\
n=451\end{array}$ & $\begin{array}{c}\text { V/O } 2008 \\
n=27649\end{array}$ & $\begin{array}{c}G 09-15 \\
n=418\end{array}$ & $\begin{array}{l}\text { V/O } 2015 \\
n=23273\end{array}$ \\
\hline EcoTF* & 95,6 & 90,6 & 98,8 & 90,2 \\
\hline HIPV grave‡ & 9,3 & 7,9 & 8,5 & 7,1 \\
\hline LPV§ & 3,8 & 3 & 5 & 2,8 \\
\hline Observação oftalmológica & 64,1 & 73,6 & 76,1 & 75,2 \\
\hline ROP grave nos RN observadosIl & 7,6 & 7,4 & 4,7 & 5,5 \\
\hline Cirurgia ROP/anti-VEGFI & $0,9 / \mathrm{na}^{* *}$ & $4,2 / \mathrm{na}^{* *}$ & $0,7 / 1$ & $2,1 / 1,2$ \\
\hline
\end{tabular}

* Eco TF: ecografia transfontanelar; ₹ HIVP grave: hemorragia intra e periventricular graus 3 e 4; § LPV: leucomalácea periventricular; II ROP grave: retinopatia da prematuridade graus 3 e 4 ( G01 - $08 n=289$; G 09 - $15 n=318$ ); T Cirurgia ROP/ VEGF: cirurgia da retinopatia da prematuridade/ administração de bevacizumab ou outra droga anti-VEGF; ** na: não aplicável 
Tabela 5 - Patologia gastrointestinal e malformativa

\begin{tabular}{|c|c|c|c|c|}
\hline$\%$ & $\begin{array}{c}G 01-08 \\
n=451\end{array}$ & $\begin{array}{l}\text { V/O } 2008 \\
n=27649\end{array}$ & $\begin{array}{c}G 09-15 \\
n=418\end{array}$ & $\begin{array}{c}\text { V/O } 2015 \\
n=23273\end{array}$ \\
\hline NEC* & 2,9 & 6,0 & 5,5 & 4,5 \\
\hline Cirurgia NEC & 0,9 & 2,5 & 2,6 & 2,9 \\
\hline Perfuração GI‡ & 1,1 & 2 & 1,7 & 1,5 \\
\hline Malformações major & 3,8 & 4,4 & 3,1 & 4,8 \\
\hline
\end{tabular}

* NEC: enterocolite necrosante; ₹ GI: gastrointestinal

Tabela 6 - Na altura da alta hospitalar

\begin{tabular}{|c|c|c|c|c|}
\hline & $\begin{array}{c}\text { G } 01-08 \\
n=451\end{array}$ & $\begin{array}{c}\text { V/O } 2008 \\
n=27649\end{array}$ & $\begin{array}{c}G 09-15 \\
n=418\end{array}$ & $\begin{array}{c}\text { V/O } 2015 \\
n=23273\end{array}$ \\
\hline Alta para casa $(\%)$ & 73,6 & 74,9 & 79,2 & 74,3 \\
\hline Transferência $(\%)^{*}$ & 10,6 & 10,3 & 8,4 & 11,4 \\
\hline Falecido (\%) & 15,5 & 15,8 & 12,4 & 13 \\
\hline Peso na alta (média g \pm desvio padrão/mediana g) & $2027 \pm 674 / 2115$ & 2200 & $2107 \pm 618 / 2150$ & 2317 \\
\hline PC na alta (média $\mathrm{cm} \pm$ desvio padrão/mediana $\mathrm{cm}$ ) $\ddagger$ & $32 \pm 3,3 / 32,5$ & 31,2 & $31,8 \pm 3,3 / 32,5$ & 31,5 \\
\hline LOS (média dias \pm desvio padrão / mediana dias)§ & $50,4 \pm 36 / 44$ & 52,9 & $55 \pm 32 / 53$ & 56,5 \\
\hline
\end{tabular}

* Transferência: transferência para outro hospital para continuação de cuidados médicos; ₹ PC: perímetro cefálico G01 - 08 n = $135 /$ G09 - 15 n = 402 ; $\S$ LOS: tempo de internamento no nosso hospital considerando todos os RN (alta para o domicilio, transferidos e óbitos)

essa altura $629 \mathrm{RN}$, dos quais $14,3 \%$ com aleitamento materno exclusivo, 56,3\% com leite adaptado e $29,4 \%$ com formas mistas de aleitamento.

\section{DISCUSSÃO}

É muito importante para um serviço de Neonatologia conhecer as características da população que assiste, aferir a qualidade dos seus cuidados e promover a sua divulgação entre os pares. Um registo como o de Vermont Oxford permite identificar os pontos fortes e fracos da prática assistencial dos hospitais participantes.

Este trabalho permitiu fazer a reflexão sobre os dados da nossa UCIN e compará-los com os dados de um dos maiores registos mundiais, a que a nossa UCIN também pertence. Inevitavelmente várias foram as limitações deste trabalho. Não tivemos acesso aos dados individuais de cada RNMBP do registo do V/O e por conseguinte não nos foi possível a realização de análise estatística comparativa. As unidades de neonatologia notificadoras do V/O são certamente heterogéneas em termos de cuidados prestados. $O$ facto de termos considerado só os RNMBP nascidos no hospital onde foram tratados permite excluir unidades menos diferenciadas e enviesamentos como o transporte ex-útero do recém-nascido. Optámos por incluir todos os hospitais com o mesmo nível de diferenciação do nosso. Neste capítulo poderíamos incluir somente o grupo de hospitais Europeus mas isso iria diminuir muito o número total de RNMBP, os valores seriam menos fiáveis e não nos estaríamos a comparar com práticas assistenciais neonatais globais.

Verificámos, pelos dados somatométricos e de idade gestacional, que a nossa população de RNMBP é muito sobreponível ao global do $\mathrm{V} / \mathrm{O}$, sendo a diferença mais notória a percentagem de RN LIG (28\% vs $23,3 \%)$ mas que poderá ser explicada pela utilização de tabelas americanas, podendo ocasionar um fator de enviesamento.
Podemos considerar sobreponíveis as taxas de vigilância da gravidez e de cesarianas, sendo mais frequente a realização de indução maturativa no nosso hospital. Em relação à gestação verifica-se que o nosso hospital tem uma taxa maior de gestações gemelares.

Verificámos que o Serviço acompanhou a evolução que a Neonatologia foi tendo ao longo dos anos ${ }^{7}$ no que concerne, por exemplo, a práticas e terapêuticas respiratórias menos invasivas e de promoção da amamentação materna do RNMBP. É interessante documentar a evolução das práticas neonatais em dois capítulos importantes: a administração de corticoides como terapêutica da displasia bronco-pulmonar em que nos primeiros anos de registo essa prática era ainda incentivada, tendo havido nos últimos anos uma inflexão importante na sua prescrição. ${ }^{8}$ Também a administração de surfactante profilático tem vindo a decrescer com orientações mais estritas nos últimos tempos, nomeadamente entre nós com a norma da Direção-Geral de Saúde publicada em 2012.9,10

Um dos aspetos negativos que é urgente melhorar é a taxa de infeção associada aos cuidados de saúde em que há na nossa série um desvio notório relativamente aos resultados da rede com relevância para a sépsis a estafilicocos coagulase negativos $(5,8 \%$ no primeiro período e aumento muito importante para $19,1 \%$ no segundo período temporal). Estes números terão de ser estudados e identificadas as razões para tal aumento, nomeadamente porque é que a sua evolução é tão distinta dos valores da rede V/O. Também as sequelas neurológicas, o controle da hipotermia na admissão à UCIN e a administração pré-parto de sulfato de magnésio são aspetos a melhorar.

Verificaram-se $14 \%$ de óbitos dos recém-nascidos internados correspondendo a $15,5 \%$ e $12,4 \%$ nos dois grupos temporais e no registo $\mathrm{V} / \mathrm{O}$ de $15 \%$ e $13,8 \%$ em 2008 e 2015 , respetivamente. Os nossos recém-nascidos na altura 
da alta tinham um peso sensivelmente menor e um tempo de internamento e valores de perímetro cefálico (que variou entre os 31 e os $32 \mathrm{~cm}$ em todos os grupos) sobreponíveis.

Verificamos, por este registo, que os RNMBP são atualmente uma população heterogénea. É completamente distinto o nível de assistência neonatal necessária a um RN com 600 ou com 1400 g, por exemplo, assim como a sua mortalidade e morbilidade. Assim nunca será de mais enfatizar a necessidade de praticar uma perinatologia de excelência evitando o mais possível o parto muito pré-termo. Embora no nosso estudo só tenhamos incluído RNMBP nascidos no Centro Hospitalar nunca será demais referir a necessidade de promover uma atempada transferência in útero para um hospital perinatal diferenciado em caso de ameaça de parto muito aquém do termo.

Muitas das sequelas da prematuridade, sobretudo nos mais imaturos, não são evidentes na altura da alta da UCIN tal como foi bem estabelecido nos estudos EPICure. É sabido que mesmo RNMBP sem complicações durante o internamento podem ter mais tarde múltiplos problemas que terão de ser abordados atempadamente para a sua minimização. ${ }^{11-13}$ Assim é evidente a necessidade de um seguimento a longo prazo destas crianças, que geralmente é feito até à entrada na escolaridade obrigatória, para se conseguir de facto aferir a qualidade da prática médica exercida no nosso Serviço de Neonatologia.

\section{CONCLUSÃO}

Com este trabalho aferimos com satisfação muitos dos nossos dados e ficámos alerta para a necessidade de melhorar certos indicadores fundamentais para a melhoria dos cuidados prestados aos nossos RNMBP. É fundamental em Neonatologia fazer-se a reflexão e benchmarking do trabalho realizado e seus resultados. Esperamos que outras unidades portuguesas possam beneficiar da divulgação destes dados, fazer o estudo comparativo com os seus próprios números, dar-nos o seu feedback, e se oportuno discuti-los, em prole da melhoria dos resultados da Neonatologia Portuguesa

\section{AGRADECIMENTOS}

À Abbott (atualmente Abbvie) pelo financiamento da participação na rede V/O de 2001 a 2014. Ao centro hospitalar pelo financiamento do ano de 2015. A Lincoln Justo da Silva por ter sempre incentivado o estudo dos dados do V/O e a sua comparação entre UCIN portuguesas.

\section{PROTEÇÃO DE PESSOAS E ANIMAIS}

Os autores declaram que os procedimentos seguidos estavam de acordo com os regulamentos estabelecidos pelos responsáveis da Comissão de Investigação Clínica e Ética e de acordo com a Declaração de Helsínquia da Associação Médica Mundial.

\section{CONFIDENCIALIDADE DOS DADOS}

Os autores declaram ter seguido os protocolos do seu centro de trabalho acerca da publicação de dados.

\section{CONFLITOS DE INTERESSE}

Os autores declaram não ter conflitos de interesses relacionados com o presente trabalho.

\section{FONTES DE FINANCIAMENTO}

Este trabalho não recebeu qualquer tipo de suporte financeiro de nenhuma entidade no domínio público ou privado.

Changes in the use of postnatal steroids for bronchopulmonary dysplasia in 3 large neonatal networks. Pediatrics. 2006;118:e1328-35.

9. Sweet DG, Carnielli V, Greisen G, Hallman M, Ozek E, Plavka R, et al. European Consensus Guidelines on the Management of Respiratory Distress Syndrome - 2016 Update. Neonatology. 2017;111:107-25.

10. Direção-Geral da Saúde. Administração de surfactante pulmonar no síndrome de dificuldade respiratória do recém-nascido. Norma n 012/2012. Lisboa: DGS; 2012.

11. Johnson S, Strauss V, Gilmore C, Jaekel J, Marlow N, Wolke D. Learning disabilities among extremely preterm children without neurosensory impairment: Comorbidity, neuropsychological profiles and scholastic outcomes. Early Hum Dev. 2016;103:69-75.

12. Johnson S, Kochhar P, Hennessy E, Marlow N, Wolke D, Hollis C. Antecedents of attention-deficit/hyperactivity disorder symptoms in children born extremely preterm. J Dev Behav Pediatr. 2016;37:285-97.

13. Costeloe KL, Hennessy EM, Haider S, Stacey F, Marlow N, Draper ES. Short term outcomes after extreme preterm birth in England: comparison of two birth cohorts in 1995 and 2006 (the EPICure studies). BMJ. 2012;345:e7976. 